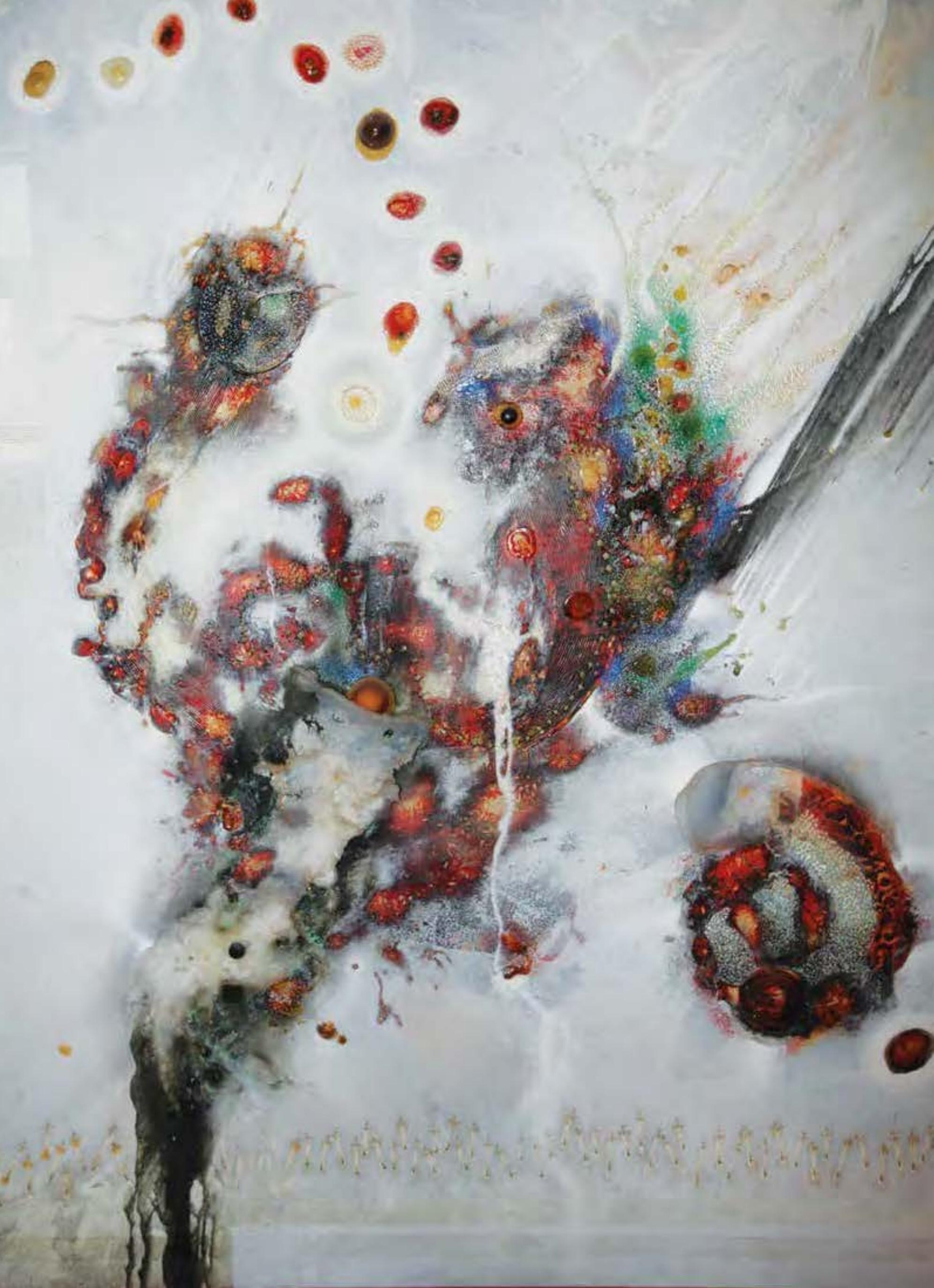




\section{Poema Guadalajara}

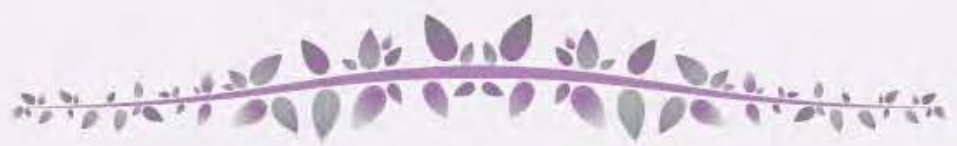

Eres hermosa Guadalajara,

donde el mariachi y su charrería

envuelven el folkfore de la tierra tapatía.

Eres flores y monumentos,

y sutil esencia de provincia,

eres mágica en tu gente

que es cálida porque es mexica.

Tus grandes torres de catedral

te ven crecer más cada día,

y las incontables fuentes, que son tu adorno,

chorrean y alegran tus avenidas.

Eres Cabañas y un Degollado

que albergan música e historia viva.

Tus tradiciones y grandes fiestas,

tu alfareria y vidrio soplado,

son un embrujo al extranjero

que gusta de todo lo que es mexicano.

Guadalajara, Guadalajara,

T'ú, eres mi tierra, yo, soy tu vida. 


\title{
2015: Auge Laboral en la Región Metropolitana Guadalajara
}

\author{
${ }^{1}$ JOSÉ MARÍA PARRA RUIZ \\ 2 EMILIA GÁMEZ FRÍAS \\ Recibido 31.05.16 / Aprobado 30.06.16
}

\begin{abstract}
RESUMEN. En 2015, la población de la Región Metropolitana Guadalajara (RMG), registra un auge ocupacional. Más de 73 mil nuevas ocupaciones fueron generadas por nueve ramas de actividad económica. Sin embargo, estas ocupaciones resultan insuficientes para cubrir las necesidades que demanda la población en edad de trabajar (15 y más años de edad). Cabe señalar que el mayor crecimiento lo experimenta la fuerza de trabajo en términos generales y por rama de actividad económica donde labora. Pero a la vez, dicha fuerza de trabajo incrementó su compra-venta bajo disímbolas condiciones de precariedad. Por lo tanto se observa que el mayor nivel de ocupación se realiza bajo condiciones de precariedad laboral (en aras de incrementar ganancias) lo cual contraviene el postulado constitucional establecido en el artículo $123^{3}$. Ello, conlleva a incrementar la inestabilidadheterogeneidad laboral en la población de la RMG, principalmente de la fuerza de trabajo. Cabe señalar que el análisis de la información estadística: Encuesta Nacional del Empleo y la Ocupación (ENOE, segundo trimestre de cada año del periodo) ${ }^{4}$, se fundamenta en la perspectiva teórica conceptual de la economía política y la sociología del trabajo.
\end{abstract}

Palabras Clave: inestabilidad-heterogeneidad laboral; precariedad laboral: auge laboral.

ABSTRACT. In 2015, the population of Guadalajara metropolitan area (RMG) recorded an occupational boom. More than 73,000 new jobs $w$ ere generated by nine branches of economic activity. However, these occupations are insufficient to meet the needs demanded by the population of working age (15 and more years old). It should be noted that the highest growth experienced workforce in general and by branch of economic activity where he works. But at the same time that labor force increased its buying and selling dissimilar under precarious conditions. Thus it is seen that the highest level of occupation is performed under conditions of job insecurity (in order to increase profits) which contravenes the constitutional postulate laid down in Article 123. This leads to increased instability-heterogeneity labor in the population RMG, mainly from the workforce. It should be noted that the analysis of statistical information: national survey of employment and occupation (ENOE second quarter of each year of the period), is based on the conceptual theoretical perspective of political economy and sociology of work.

Keywords: labor instability-heterogeneity; job insecurity: employment boom.

\section{Introducción}

En el pensamiento social priva un extenso e intenso debate sobre el mercado de trabajo. En este debate se expresan diversas concepciones teórico-conceptuales-metodológicas. Cabe destacar las concepciones que formulan una crítica al enfoque neoclásico, toda vez que fundamentan, con evidencia empírica, que el mercado de trabajo tiende a ser inestable y heterogéneo en todo tiempo, espacio laboral y lugar geográfico (Rojas y Salas, 2008; Sotelo, 2012; Antunes, 2012; Gómez, 2007). Este rasgo de inestabilidad-heterogeneidad, constituye una herramienta heurística de la mayor importancia en el examen del mercado de trabajo de los países desarrollados y, también, no desarrollados. En América Latina el examen de la inestabilidad-heterogeneidad del mercado de trabajo es realizado, en los últimos años, en el marco de concepciones como la segmentación y precariedad laboral (Hualde y Guadarrama, 2012; Alves, 2012; Sotelo, 2012).

El primer enfoque, segmentación, reconoce las diferencias y variaciones muy acentuadas que existen en las condiciones de empleo y trabajo de los asalariados proponiendo examinar el mercado de trabajo a través de segmentos: mercados internos (configurado mediante normas y reglas, para los ascensos, por ejemplo) y externos (mercados dualistas: primario-secundario; triple segmentación: administrativos, técnicos e ingenieros, etc.). A esta concepción se incorporan otros enfoques como el

1. Dr. En Ciencias Sociales por El COLEF, Profesor-Investigador, Departamento de Estudios Regionales-INESER, UDG. jparra@cucea.udg.mx

2. Maestra en Economía y Negocios por la Universidad de Guadalajara. Profesor-Investigador, Departamento de Estudios Regionales-INESER, Universidad de 2. Guadalajara, emigamez@ cucea.udg.mx

3. En el Título Sexto Del Trabajo y de la Previsión Social del artículo 123 constitucional expresa:”Toda persona tiene derecho al trabajo digno y socialmente útil; al efecto, se promoverán la creación de empleos"... y en su apartado VI señala: "Los salarios mínimos generales deberán ser suficientes para satisfacer las necesidades normales de una familia, en el orden material social y cultural, y para proveer la educación obligatoria de los hijos" Es decir, se considera vital garantizar empleo para toda la población, así como una remuneración que permita al trabajador y a su familia reproducirse en condiciones decentes.

4. Seleccionamos el segundo trimestre, de cada año, en razón de que resulta ser el que en menor medida se encuentra afectado por las fluctuaciones estacionales de la actividad económica; así como porque, en dicho trimestre, se utiliza una versión ampliada del Cuestionario ENOE. 
de la segregación laboral por género, mercados de trabajo locales y redes sociales (Pries, 2000).

Por su parte la concepción de precariedad laboral, parte de reconocer que un mayor número de segmentos de trabajadores, asalariados y por cuenta propia, experimentan un deterioro en sus condiciones laborales examinadas en diferentes dimensiones. Especialistas del tema subrayan la ambigüedad y polisemia del concepto. asi como, el carácter mutante y gradual de la precariedad. Con el fin de solventar dicha situación, Guadarrama et al (2014), proponen identificar y operacionalizar en estudios de caso y comparativos, cuatro dimensiones: nivel salarial; tipo de contrato; acceso a servicios de salud y prestaciones laborales, entre otros indicadores.

Sobre la base del examen de estas cuatro dimensiones espigan una serie de estudios que se aproximan a conocer y explicar el deterioro de las condiciones de compra-venta de fuerza de trabajo que imperan en el mercado de la fuerza de trabajo, durante todo el proceso de acumulación de capital impulsado por la vía neoliberal.

Entre los hallazgos vertidos por la serie de estudios de caso, a escala de América Latina, pais, y territorio subnacional (regiones urbanas y rurales), destacan:

1. La polarización permanente del mercado de trabajo (segmentos de fuerza de trabajo asalariada con distintos niveles de precariedad laboral y segmentos sin precariedad laboral).

2 Procesos de precarización: mayores y menores analizados por cada una de las cuatro dimensiones objetivas.

3. Bajos niveles salariales como rasgo permanente de la precariedad laboral en América Latina, México y en todo espacio urbano y rural. En suma, todo espacio territorial muestra en mayor o menor medida rasgos definitorios de precariedad laboral.

En México, además de los estudios nacionales destacan los estudios comparativos de la fuerza de trabajo a escala urbana. En esta vertiente destacan los estudios comparativos de la precariedad que registra Guadalajara, Ciudad de México y Monterrey (García, 2009 y 2010; Rubio, 2010; Gaxiola, 2013). En distintos trabajos comparativos, los cuales dicho sea de paso resultan escasos, se afirma que la precariedad laboral en la metrópoli de Guadalajara se encuentra fuertemente arraigada en la estructura ocupacional al igual que en la ciudad de México. Sin embargo, se concluye que Guadalajara se encuentra por arriba del nivel de precariedad que registra Monterrey y casi al mismo nivel de la ciudad de México (Gaxiola, 2013).
Esta valoración, se sustenta en el análisis de las cuatro dimensiones objetivas de la precariedad laboral señaladas. Sin embargo, no se da cuenta del segmento de trabajadores que laboran bajo condiciones no precarias. Por lo anterior, parece necesario, por una parte, actualizar el examen del mercado de trabajo de la RMG, en especial lo correspondiente al nivel de ocupación y, de empleo; por otra, analizar las condiciones que prevalecen en la compraventa de fuerza de trabajo que prevalecen en el mercado y las configuraciones laborales que se producen: precarias y no precarias. Ambas consideraciones, se incorporan en el presente trabajo.

En dicha trayectoria de estudios laborales se inscribe el presente trabajo. El argumento central es que la población de la RMG, al cabo de un año de crisis laboral, experimenta en 2015, un crecimiento en el nivel de ocupación, el cual, si bien revierte la crisis laboral de 2014, es insuficiente para cubrir las necesidades que precisa dicha población regional. Simultáneamente, se observa, por una parte, una disminución marginal en el nivel de desempleo; por otra, que la fuerza de trabajo no registra una mejoría sustantiva en las condiciones de su compra por parte de los distintos empleadores. Ello, en el contexto de un exiguo crecimiento económico, y un estancamiento de la participación de la población en el mercado de trabajo (Gráfico $\mathrm{N}^{\circ} 1$ ).

\section{Gráfico $N^{\circ} 1$. Tasas de crecimiento y participación laboral en el mercado de trabajo, 2005-2015}

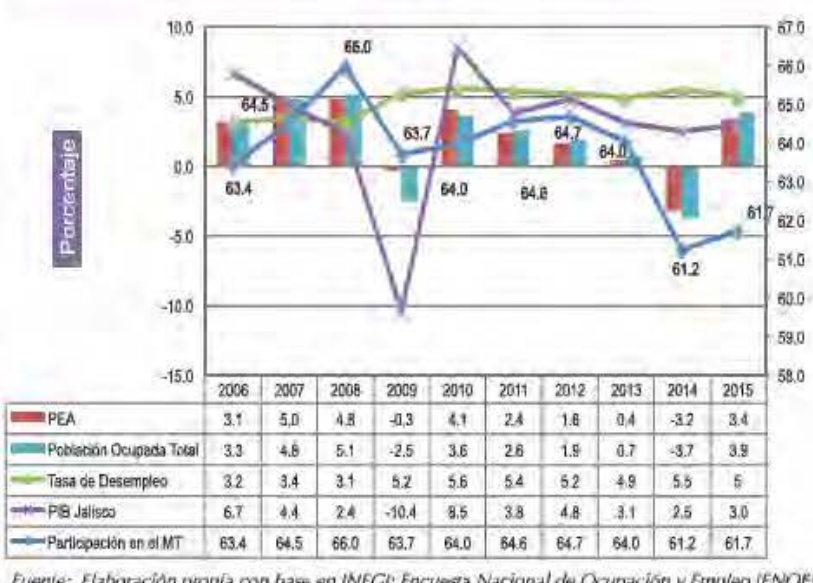

\section{Meiodologia}

El propósito de este trabajo es ofrecer evidencia estadística, por una parte, sobre la inestabilidad en el nivel de ocupacion a escala regional $\mathrm{y}$, sus impactos heterogéneos en los sujetos laborales que configuran la estructura ocupacional; por otra, evidencia sobre la inestabilidad laboral que registra la fuerza de trabajo (principal sujeto laboral) en cada actividad económica, asi 
como, las heterogéneas condiciones de su compra-venta que imponen los empleadores de las distintas ramas de actividad económica. Ello, con el fin de establecer si el incremento en el nivel de la ocupación, obtenido en 2015 , es suficiente para cubrir las necesidades que demanda la población de la RMG, así como, si éste incremento de puestos laborales tiende a mejorar las condiciones de compra-venta de la fuerza de trabajo que se agudizaron con la crisis laboral de 2014.

Con la finalidad de cumplir con dichos objetivos, por una parte, se operacionalizaron algunas concepciones teórico-conceptuales-metodológicas de la economia política y de la sociologia del trabajo. Asimismo, se procesó y analizó información estadística propia de la Encuesta Nacional del Empleo y Ocupación (ENOE), correspondiente al segundo trimestre de cada uno de los años del periodo de estudio: 2005-2015.

El examen se basa en las tasas de participación y de crecimiento promedio anual, así como en valores absolutos sobre los incrementos y pérdidas que registra cada uno de los indicadores utilizados, con la finalidad de ofrecer evidencia sobre el estado que guarda la estructura y la dinámica del empleo, asi como, las condiciones de compra-venta de la fuerza de trabajo en el año 2015 , comparativamente con 2014 y, en general con respecto al periodo 2005-2015.

Con base en lo anterior, se expone la taxonomia de las trayectorias de precariedad y no precariedad laboral por cada uno de estos dos segmentos que configuran dicho mercado.

\section{Discusión de resultados}

\subsection{Situación de la ocupación}

En 2015 la población ocupada de la (RMG), experimentó el mayor incremento de los últímos cinco años ${ }^{5}$. En dicho año, 73,489 nuevos trabajadores se incorporaron a desempeñar su labor en distintas actividades económicas; sin embargo, simultáneamente, otros 5,608 trabajadores perdieron su ocupación. En consecuencia, la diferencia de ambos trabajadores (nuevos y desocupados), asciende a $67,881^{6}$ nuevas plazas laborales. Cabe señalar que, con respecto a esta última cantidad, el $59.5 \%$ de las nuevas plazas laborales las ocupan hombres y, el restante $40.5 \%$ mujeres. Dichas proporciones, se asemejan a la participación porcentual que hombres $(59.3 \%)$ y mujeres
$(40.7 \%)$ presentan respecto al número total de la población ocupada en 2015 (Gráfico No 2).

Gráfico $N^{\circ}$ 2. RMG: tasas de participación y de erecimiento anual de la población ocupada por sexo, 2005-2015

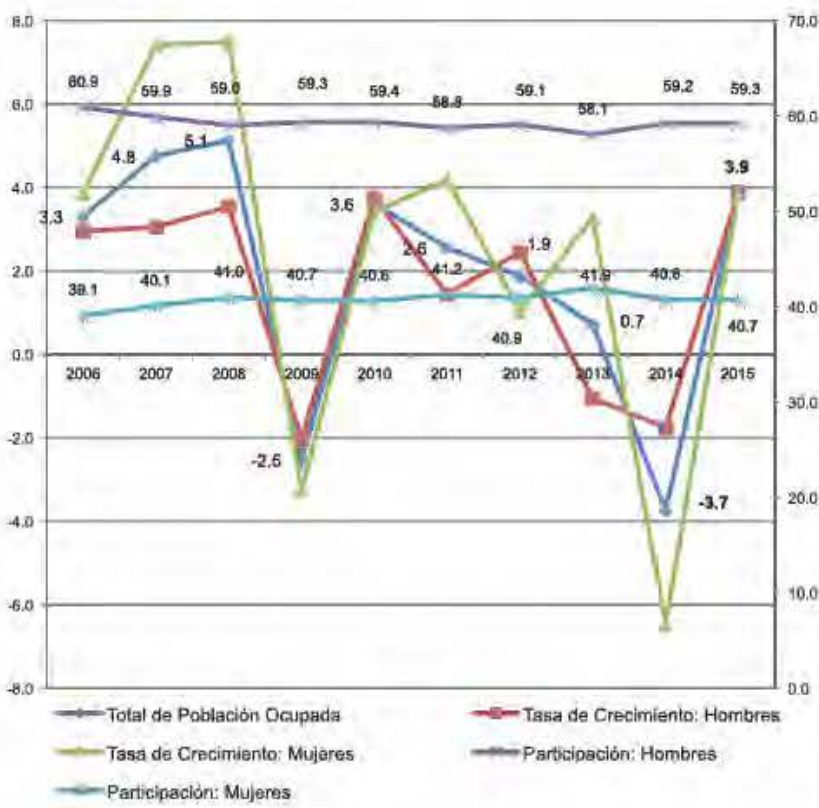

Fuerte- Elabarabión prapia cón base en INECG): Encuesta Narional de Ocupadien y Emplea (ENOE)

De acuerdo a lo anterior, el incremento de población ocupada experimentado en 2015 , configura una dinámica de $3.9 \%$ (Gráfico $N^{\circ} 2$ ), respecto de 2014 (solo superada por el $5.1 \%$ registrado en 2008). Este dinamismo de $3.9 \%$, también lo registra, en 2015 , el incremento de nuevos empleos que ocupan hombres (el mayor del último decenio) y mujeres (dicha dinámica les permite superar su participación de los últimos tres años, misma que resulta inferior respecto a otros años) (Gráfico $N^{\circ} 2$ ). Lo anterior, pone en evidencia que, los hombres por primera vez, en los últimos diez años, registran su mayor dinámica en la obtención de nuevas ocupaciones en la RMG. Algo que las mujeres registran dos años antes del estallido de la crisis de 2009, pero con una dinámica de casi $8 \%$ (Gráfico $\mathrm{N}^{\circ} 2$ ), Por consiguiente, la dinámica participación de la mujer en el mercado de trabajo constituye un importante factor explicativo sobre el comportamiento del empleo en esta porción territorial de Jalisco, México.

Este incremento ocupacional, contribuye: 1) al descenso de la población desempleada (aunque la tasa de desempleo registra un nivel de $5 \%$, como ocurre desde

5. Cabe seîalar que este incremento de empleos que experimenta la población de la RMG, resarce, paradójicamente, en la misma cantidad la pérclida ocurrida en 2014 , la cual dicho sea de paso, configura la crisis laboral más profunda del presente siglo en dicha porción territonal. Para unia amplia información sobre la crisis laboral, de 2014, en la RMG véase Parra y Gámez (2014).

6. Esta cantidad de nueva población ocupada, se coloca, en la última decada, en la tercera posición solo por debajo de los incrementos registrados en 2008 (83, 666) y 2007 (73,961). 
2009); 2) a que la PEA experimente un incremento (en la misma proporción que la registrada en 2007 y, solo inferior al obtenido en 2008); 3) a que la participación de la población en el mercado de trabajo (prácticamente estancado con respecto a 2014), registre un ligero incremento, aunque, distante al alcanzado en 2008 (Gráfico $\left.\mathrm{N}^{\circ} 1\right)$.

Frente a la evidencia estadística de un incesante aumento y disminución de la población ocupada (en términos generales), parece importante incorporar otro matiz analítico que permita identificar los impactos diferenciales que enfrentaron los sujetos laborales como efecto del aumento y/o disminución en su nivel de ocupación. Para tal propósito, se establece la interrogante ¿Qué sujetos laborales incrementaron y disminuyeron su participación en el nivel de su ocupación y que consecuencias enfrentan?

\subsection{Los sujetos laborales}

Frente al aumento y disminución en el nivel de ocupación, el objetivo del presente apartado es analizar, por una parte, la participación que adquieren los trabajadores en la estructura ocupacional de la RMG; por otra, la compra y despido de fuerza de trabajo que realizan los empresarios, de las diferentes ramas de actividad económica, del trabajador que registra la mayor participación en la estructura ocupacional. Ello, con el fin de identificar la inestabilidad-heterogénea que enfrentan, por una parte, la población ocupada, por otra, la fuerza de trabajo en cada uno de sus espacios laborales.

\subsubsection{Distribución y contribución de los incrementos y pérdidas en la población ocupada}

Como se señala en el apartado anterior, en 2015, más de 73 mil hombres y mujeres se sumaron como nueva población ocupada en la RMG. Esta cantidad de nuevas ocupaciones, espiga como la tercera más importante de la última década ( $13.8 \%$ por debajo de la registrada en 2008 y $4.5 \%$ menor a la obtenida en 2007). Cabe señalar que, dicho incremento contribuye para que el total de la población ocupada, por segunda ocasión, registre su máximo histórico ${ }^{7}$.

En lo correspondiente a la estructura ocupacional, se presenta lo siguiente. Del número total de nuevas ocupaciones, el $72.7 \%(53,410)$ corresponden a la fuerza de trabajo. Este incremento, permite que dichos trabajadores registren el mayor número de empleos, de toda su historia en la RMG: 1,349, 346 hombres y mujeres (en las proporciones signadas en el Gráfico $\mathrm{N}^{\circ} 2$ ), superior en $2.1 \%$ al registrado en 2013. Es decir, en 2015, la fuerza de trabajo que labora en la RMG obtuvo su máximo histórico de empleos subordinados y remunerados mediante la compraventa de su capacidad de trabajo. A su vez, el incremento de empleos que experimenta la fuerza de trabajo le permite revertir en más del $100 \%$ las pérdidas registradas un año antes. Por último, su dinámico crecimiento de $4.1 \%$, en 2015 respecto al año anterior, es superior al registrado en los últimos cuatro años (Gráfico $\mathrm{N}^{\mathrm{o}} 3$ ).

Ensegundo término, destaca el incremento experimentado por los trabajadores por cuenta propia: 26\% (19,105 nuevas ocupaciones). Si bien es cierto que esta cantidad de nuevos trabajadores prorrumpe como el segundo mayor incremento de la última década (7.1\% inferior con respecto a 2009), no logra revertir la pérdida experimentada en 2014 de 40,445 trabajadores (Gráfico $N^{o} 3$ ). Cabe señalar, que este tipo de trabajadores independientes registran, casi siempre, una relación inversa respecto a la ocupación de la fuerza de trabajo: cuando esta última se incrementa los primeros disminuyen y viceversa. Por lo tanto, los trabajadores no remunerados constituyen una reserva de población importante para que en ciertas coyunturas logren emplearse como fuerza de trabajo remunerada. En tercer término destaca, el incremento de los empleadores o sujetos generadores de empleo aunque su crecimiento es mínimo (1\%), en 2015 (Gráfico $\mathrm{N}^{\mathrm{o}} 3$ ), es importante considerarlo toda vez que de alguna manera explica el incremento en la compra de fuerza de trabajo y en la contratación de trabajadores por cuenta propia en relación directa con la actividad económica que impulsan.

Por su parte los trabajadores no remunerados, en 2015 , vuelven a registrar otra considerable pérdida de empleos: 5,608. Cabe señalar que en los últimos cinco años, este tipo de trabajador, registra una consistente pérdida de empleos (Gráfico No 3 ).

Por todo lo anterior, en 2015, la estructura ocupacional experimenta un comportamiento contrario al del año anterior: tres tipos de trabajadores experimentaron incrementos en su nivel de ocupación y solo uno registra pérdidas. Esto demuestra el comportamiento ocupacional de inestabilidad-heterogénea que enfrenta la población de la RMG, como consecuencia de la inestabilidad económica, social, política, etc. que enfrenta el país, así como producto de los requerimientos que precisa el proceso de acumulación de capital, como también, por la falta de un compromiso social por parte de la clase política y empresarial y, por su ineficiencia e ineficacia respecto al proyecto depredador que impulsan en lo económico como en lo productivo en las últimas tres décadas.

7. En 2013 la población ocupada total, en la RMG, ascendió a 1 millón 821 mil. Misma cantidad registrada dos años después. 
Gráfico $\mathrm{N}^{\circ} 3$. Incrementos y pérdidas de la ocupación de los sujetos laborales segón su posición en la estructura ocupacional, 2005-2015

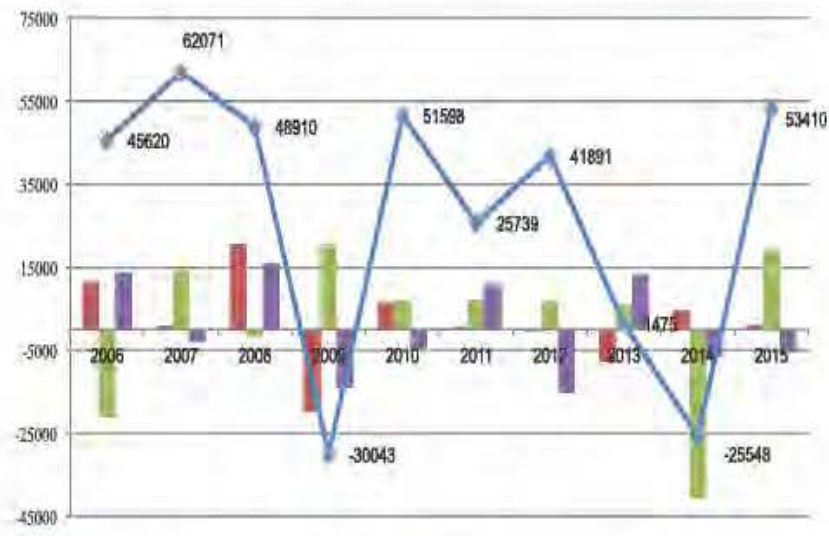

$=$ Empleadores $=$ PorCuenta Propia $=$ No Remunerados $\Rightarrow$ Fuera de Trabajo

Fuente: Elaboraciön propia con hase en INEG): Encuecta Nacional de Ocujpaciân y Empleo (ENOE)

3.2.2. Participación de la población ocupada en la estructura ocupacional

Sin duda el aumento de la fuerza de trabajo ocupada, en 2015, solo, impacta marginalmente su participación en la estructura ocupacional. Los nuevos empleos $(53,410)$, incrementaron dicha participación en 0.2 puntos porcentuales, respecto de 2014, para alcanzar su máximo histórico de $74.1 \%$ (Gráfico No 4). Es decir, por cada 10 trabajadores ocupados en la RMG, 7.4 son fuerza de trabajo. Cabe señalar que este nivel histórico de su participación en la estructura ocupacional, permite que la fuerza de trabajo ocupada de la RMG consolide la segunda posición respecto de las principales ciudades del pais: 6.4 puntos porcentuales por debajo de la participación de la fuerza de trabajo de Monterrey, Nuevo León; y, 1.9 puntos porcentuales por encima de la Ciudad de México. Asimismo, la participación de la fuerza de trabajo de la RMG se ubica en 6.2 puntos porcentuales por arriba del promedio registrado a escala nacional, en 2015. De acuerdo a lo anterior, la fuerza de trabajo de la RMG constituye una variable explicativa importante de considerar en el análisis de la generación y pérdida de empleo, así como de las condiciones que prevalecen para su compra-venta.

En segundo término, destaca la participación de los trabajadores por cuenta propia. Los nuevos trabajadores $(19,105)$, permiten que la participación de estos trabajadores independientes en la estructura ocupacional ascienda a $17.2 \%$ (incremento marginal de $0.5 \%$ en su participación respecto a 2014). No obstante lo anterior, la participación de los trabajadores por cuenta propia en 2015, espiga como la tercera más baja de la última década.
Por su parte los nuevos empleadores (empresarios), presentan un marginal retroceso en su participación porcentual en la estructura ocupacional ( -0.2 puntos porcentuales), no obstante que registran un incremento, del $1 \%$, en su número total. Por último, se encuentra la participación de los trabajadores no remunerados con $3.2 \%$ (el nivel más bajo de la última década también registrada en el 2012). Cabe señalar que estos trabajadores fueron los únicos que registran pérdidas, respecto del universo de 2014 (Gráfico No 4).

En suma, los aumentos y pérdidas de la población ocupada en 2015, no produjeron cambios importantes en la participación ni en la posición que registra cada uno de los trabajadores en la estructura ocupacional de la última década. Sin embargo, parece importante destacar el desempeño y la posición que ocupa la fuerza de trabajo en la estructura ocupacional. Con base en el hecho de que representa a las dos terceras partes de la población ocupada en la RMG, asi como también, porque su capacidad de trabajo es adquirida en el mercado y es objeto de subordinación y remuneración en todos los procesos de producción y distribución económica, reúne las cualidades suficientes para analizarlo en torno a su comportamiento en la estructura de la actividad económica impulsada en la RMG, así como, respecto a las condiciones de compraventa en el mercado.

Gráfico $N^{\circ} 4$. Sujetos laborales según posición en la estructurn ocupacioual, 2005-2015

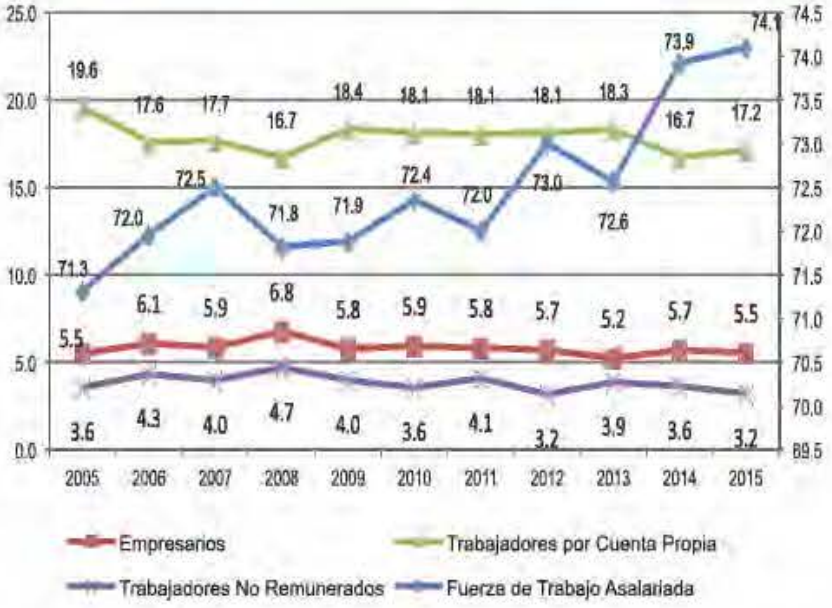

Fuenter Elaboración propua con base en INEGt: Encuesta Nacional de Ocuparía y Emplen (ENOE]

3.3 Encuentros y desencuentros entre compradores $y$ vendedores de fuerza de trabajo por actividad económica

En 2015, la fuerza de trabajo con empleo experimentó un crecimiento de $4.1 \%$ mismo que le permitió: uno, registrar su máximo histórico de empleo: 1,349, 356 
hombres y mujeres $(2.1 \%$ superior al total de fuerza de trabajo empleada en 2013); dos, superar su consistente pérdida de empleo en los últimos tres años 2015 (Gráfico $\mathrm{N}^{\circ}$ 5). Cabe señalar que, dicho incremento es la diferencia de la generación y pérdida de empleos por parte del conjunto de empleadores distribuidos en 10 de las actividades económicas que predominan en la RMG, de acuerdo a los requerimientos de su actividad y de su competencia en el mercado. Por lo que corresponde a la generación de empleos, el incremento ascendió a $80,171 \mathrm{y}$, la pérdida a 26, 761 registrando una diferencia anual de 53, 410.

La distribución del incremento en el empleo total de la fuerza de trabajo por actividad económica, es la siguiente:

a) $34.9 \%$ corresponde a la industria manufacturera $(27,454$ nuevos empleos). Esta contribución proporciona a dicha industria tres cosas:

- Alcanzar su máximo crecimiento anual en la última década: $10.1 \%$, resultado del mayor número de encuentros entre fuerza de trabajo y empleadores (Gráfico $\mathrm{N}^{\mathrm{o}}$ 5);

- Revertir los consistentes desencuentros ocurridos entre 2012 y 2014;

- Sostener su mayor participación en el universo total de la fuerza de trabajo (22.6\%); no obstante que, dicha participación se coloca como la segunda más baja de la última década (Gráfico $\mathrm{N}^{\circ}$ 6).

b) $27.4 \%$ es la contribución de la actividad de servicios sociales (21,932 nuevos empleos). Esta cantidad de nuevos empleos, también, le permitió tres cosas:

- Obtener el mayor crecimiento de nuevos empleos de todas las actividades económicas (15.5\%), pero inferior en 4.5 puntos porcentuales al registrado en 2013 (Gráfico No 5).

- Superar su crecimiento negativo de 2014 (-11\%);

- Alcanzar su máximo histórico de participación en el número total de fuerza de trabajo con empleo: $12.1 \%$ (Gráfico $\left.\mathrm{N}^{\mathrm{o}} 6\right)$.

c) $14.9 \%$ es la participación que alcanzó la industria de la construcción en la generación del total de nuevos empleos para la fuerza de trabajo $(11,954)$. Esta cantidad de nuevos empleos, conlleva tres consecuencias:

- Crecimiento del empleo de $12.9 \%$, el segundo más alto de la última década;

- Pérdida de dinamismo en su crecimiento toda vez que resulta inferior en 2.5 puntos porcentuales al registrado en 2014 (Gráfico $\mathrm{N}^{\circ} 5$ ).

- Una participación de $7.7 \%$ con respecto al número total de fuerza de trabajo empleada en la RMG (Gráfico No 6). Dicha participación, representa 0.6 puntos porcentuales superior al de 2014.

d) $12.8 \%$, constituye la aportación de los servicios profesionales, financieros y corporativos al crecimiento del empleo total $(9,065)$. Tres son las consecuencias que produce dicho incremento:

- Crecimiento de su planta laboral en $7.3 \%$

- Superar las pérdidas registradas en dos periodos anuales consecutivos (Gráfico $\mathrm{N}^{\mathrm{o}} 5$ ).

- Obtener su máxima participación histórica $(11.2 \%)$, con respecto al número total de fuerza de trabajo con empleo (Gráfico $\left.\mathrm{N}^{\circ} 6\right)$.

5) $9.2 \%(7,390)$, es la contribución de la actividad de transportes y comunicaciones al aumento que experimenta el empleo total de la fuerza de trabajo en la RMG en 2015. Dos son las implicaciones que se derivan de dicha participación, para esta actividad económica:

- Aportación de 9.6 puntos porcentuales al crecimiento total de la fuerza de trabajo empleada en esta actividad económica. La mayor aportación registrada en la última década (Gráfico $\mathrm{N}^{\circ} 5$ ).

- Incremento de 0.4 puntos porcentuales en su participación respecto al total de fuerza de trabajo empleada en 2015 (Gráfico $N^{\circ}$ 6).

6) $0.8 \%$ (648 nuevos empleos), es la participación de los restaurantes y servicios de alojamiento, con respecto al incremento total de la fuerza de trabajo de la RMG. Este aumento, produce 0.1 puntos porcentuales en su crecimiento anual el cual se convierte en el segundo más bajo de la última década (Gráfico $\mathrm{N}^{\circ} 5$ ). Asimismo, le propicia una pérdida de 0.3 puntos porcentuales en su participación con respecto al total de la fuerza de trabajo regional, comparativamente con 2014 (Gráfico No 6 ).

Por otra parte se encuentran las pérdidas de empleos en 2015: 26,761, una de las más altas en el periodo 2005 2015. La distribución de dichas pérdidas por actividad económica son las siguientes:

a) $42.4 \%$ del monto total de pérdidas de la fuerza de trabajo en la RMG $(11,353)$, corresponden al comercio (mayoreo y menudeo). Esto produce que su tasa de crecimiento anual $(0.7 \%)$, se coloque como la segunda más baja del periodo 2005-2015 (Gráfico $\mathrm{N}^{\circ}$ 5). En consecuencia, su participación porcentual de $16.8 \%$, respecto del número total de fuerza de trabajo con empleo en la RMG, resulta ser la segunda más baja de la última década y presenta una contracción de 1.5 puntos porcentuales respecto de la registrada en 2014 (Gráfico N $\left.{ }^{\circ} 6\right)$.

b) $26.8 \%(7,165)$ es la participación en el número total de pérdidas de la fuerza de trabajo, por parte de los servicios otorgados por el gobierno en sus tres niveles (federal, estatal y municipal) y de los organismos internacionales (Consulados por ejemplo) los cuales se localizan en el territorio de la RMG. Esto condujo a dichos servicios públicos, a registrar una dinámica 
laboral de $-10.0 \%$ en 2015 , el más bajo en toda la última década (Gráfico $\mathrm{N}^{\circ} 5$ ). Asimismo, propició una merma de 0.7 puntos porcentuales en su participación del total de la fuerza de trabajo con empleo, respecto de la obtenida en 2014 (Gráfico $N^{\circ}$ 6).

c) $19.0 \%$ (13,713 trabajadores), es la contribución de la actividad servicios diversos a la pérdida de fuerza de trabajo regional. Dicha contribución negativa, propicia que su crecimiento también registre un retroceso de $3.2 \%$, respecto de 2014 (Gráfico $\mathrm{N}^{\circ} 5$ ). Además, dicha perdida contribuyó para que su participación en el total de la fuerza de trabajo empleada también registrara un retroceso de 1.2 puntos porcentuales respecto a 2014 (Gráfico No 6).

d) $0.7 \%$ (1,992 empleos), es la contribución que realiza la industria eléctrica a la pérdida total de empleos en la RMG. Esta contribución negativa genera, a su vez, un crecimiento de $-2.0 \%$, es decir, 1.2 puntos porcentuales mayor al registrado en 2014 (Gráfico No 5). Sin embargo, su participación en el total regional de la fuerza de trabajo ocupada se mantiene en $0.7 \%$, como ocurre en los últimos tres años (Gráfico $\mathrm{N}^{\circ} 6$ ).

Gráfico $\mathrm{N}^{0}$ 5. Tasa de crecimiento anual de la fuerza de trabajo con empleo por actividad económica, 2005-2015

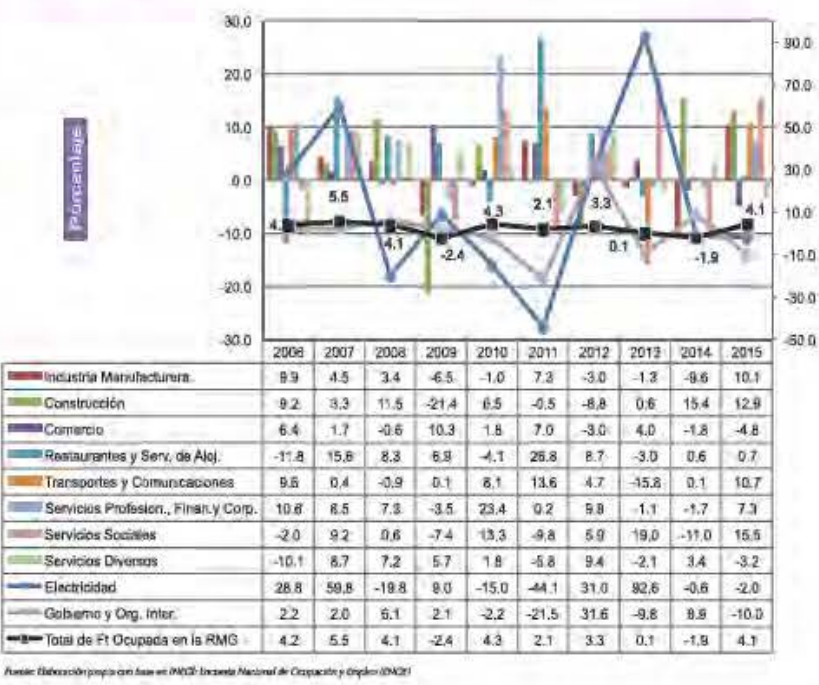

Todo lo anterior, resulta ser una clara evidencia sobre el comportamiento inestable y heterogéneo, en el tiempo y en los espacios económicos, de los encuentros y desencuentros que enfrenta la fuerza de trabajo con sus compradores (los empleadores de las diez actividades económica referidas) en la RMG. La principal evidencia de dicha situación la proporciona lo ocurrido en 2015 (auge laboral) en comparación con lo sucedido un año antes (crisis laboral). Dicho comportamiento inestable-heterogéneo en el tiempo y espacio económico registra varios matices en una doble perspectiva: interactividad economica e intractividad. Con respecto a la interactividad se pone de relieve la diferente y contrastante participación que cada una de las diez actividades económicas enumeradas, registran respecto al número total de encuentros (generación de empleo) y desencuentros (desempleo) que experimenta la fuerza de trabajo y sus empleadores en el mismo o diferentes años. En lo correspondiente a su intractividad, se destaca el inestable y heterogéneo comportamiento que registra, por una parte, las diez diferentes actividades económicas en torno a su tasa de crecimiento anual y, por otra, a la participación porcentual que presentan respecto al número total de la fuerza de trabajo empleada en la RMG.

Gráfico $N^{\circ}$ 6. Tasa de participación de la fuerza de trabajo por actividad económica, 2005-2015

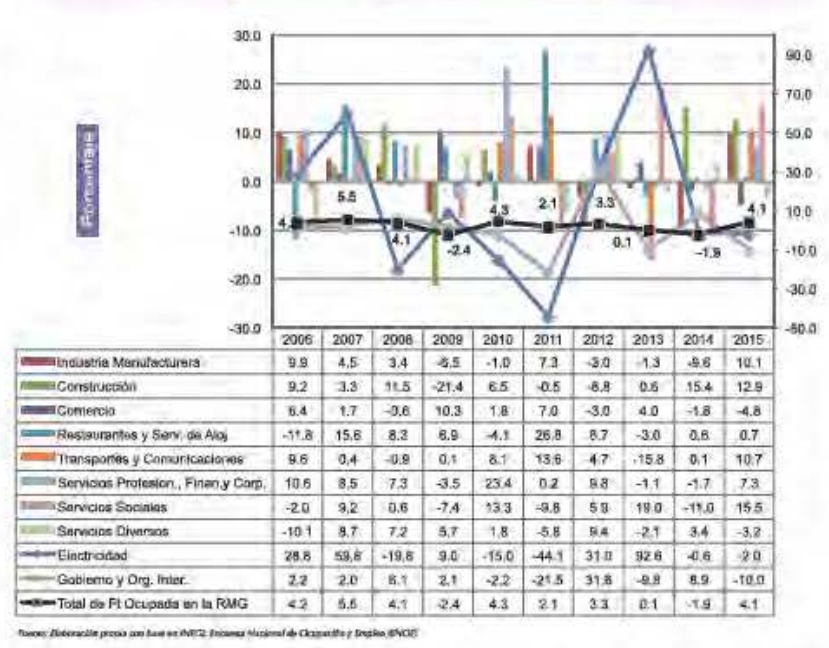

Por otra parte, se pone en evidencia que por si mismo el aumento del empleo (ejemplo, el registrado en 2015), no solo resulta insuficiente para cubrir los requerimientos que demanda, de éste, la población de la RMG, sino además, tampoco garantiza que cada una de las actividades económicas obtengan un crecimiento amual positivo y una mayor participación en el total de la fuerza de trabajo empleada. En suma, no todo crecimiento laboral es positivo toda vez que la fuerza de trabajo como es expulsada es reinsertada al empleo. Por todo lo anterior, la tendencia es a configurar un mercado de fuerza de trabajo inestable y heterogéneo con carácter altamente precario toda vez que prima la inestabilidad en la generación y en la permanencia en el empleo de la fuerza de trabajo: trabajador más importante en la estructura ocupacional de la RMG.

\subsection{Condiciones de compra-venta de la fuerza de trabajo}

En este apartado, se analizan cuatro condiciones básicas que prevalecen en la compra-venta de la fuerza de trabajo: nivel salarial, acceso a servicios de salud, prestaciones laborales y tipo de contrato. Dichas condiciones por 
actividad económica, se agruparon y delinearon cuatro configuraciones de compra-venta de fuerza de trabajo: uno, no precaria; dos, precaria alta; tres, precaria media; cuatro, precaria baja. Todo lo anterior, con el propósito de determinar si la generación y pérdida de empleos tiende por una parte, a agrupar a la mayoría de la fuerza de trabajo empleada bajo condiciones de no precariedad y, por otra, esperando que una parte minoritaria o nula labore bajo condiciones de precariedad laboral.

\subsubsection{Condiciones no precarias ${ }^{8}$}

Poco más de una cuarta parte del total de la fuerza de trabajo regional empleada (25.6\%) se compra y vende, en 2015, bajo condiciones no precarias. Es decir, del total de la fuerza de trabajo con empleo en la RMG, 25.6 laboran bajo condiciones no precarias. Bajo estas mismas condiciones laborales y por actividad económica, se encontró lo siguiente. con un promedio superior al de la RMG se encuentra la fuerza de trabajo que labora en cinco actividades económicas. Industria eléctrica (73.6\%). Del total de la fuerza de trabajo que labora en esta actividad económica, $73.6 \%$ lo hace bajo condiciones no precarias. Gobierno y organizaciones internacionales (52.4 trabajadores, del total, laboran bajo condiciones no precarias. Servicios sociales (43.3 sobre el total son trabajadores no precarios). Servicios profesionales, financieros y corporativos (32 del total laboran bajo condiciones no precarias). Industria manufacturera (28.1 del total son trabajadores no precarios).

El mismo promedio regional, lo registra la fuerza de trabajo de transportes y comunicaciones (25.6 del total de su fuerza de trabajo laboran bajo condiciones no precarias). Por debajo del promedio regional, de trabajadores no precarios se encuentra la fuerza de trabajo de cuatro actividades económicas:

- Comercio (18.1 trabajadores no son precarios).

- Construcción (14.7 no son precarios).

- Restaurantes y servicios de alojamiento (13.6 no son precarios).

- Servicios diversos (8.8 trabajadores no son precarios).

De acuerdo a lo anterior solo la industria eléctrica y los servicios del gobierno y organizaciones internacionales compran y consumen al mayor número de su fuerza de trabajo bajo condiciones no precarias. En contraste, los servicios diversos contratan al menor número de su fuerza de trabajo bajo estas condiciones. El resto de actividades, registran porcentajes diversos (Gráfico $\mathrm{N}^{\mathrm{0}} 7$ ).

\subsubsection{Condiciones de precariedad}

Con base en las cuatro condiciones de compra-venta de fuerza de trabajo, señaladas, la precariedad laboral presenta tres diferentes niveles. De suerte que, en 2015, casi tres cuartas partes de la fuerza de trabajo con empleo (74.4\%) se compró, por parte de los empleadores de las diez actividades económicas, bajo tres distintas condiciones de precariedad laboral: alta, media y baja. Es decir, del total regional de fuerza de trabajo contratada 74.4 trabajadores laboran bajo condiciones de precariedad.

\section{- Precariedad alta}

Poco más de una quinta parte del total de la fuerza de trabajo regional (20.4\%), se encuentra laborando bajo condiciones de precariedad alta. Es decir, del total de fuerza de trabajo 20.4 trabajadores laboran en condiciones de precariedad alta. Tres son las actividades económicas que registran, en su estructura ocupacional de fuerza de trabajo, promedios superiores al de la RMG: uno, servicios diversos (44.6\%); restaurantes y servicios de alojamiento (38.1\%): construcción (35.9\%). Las siete actividades restantes tienen registros inferiores al promedio regional: comercio (22.7\%); transportes y comunicaciones (14.5\%); industria manufacturera $(13.3 \%)$; servicios profesionales, financieros y corporativos (9.8\%); industria eléctrica (8.1\%); servicios sociales (6.1\%); gobierno y organismos internacionales $(2.9 \%)$. Este segmento de fuerza de trabajo constituye el polo opuesto de los trabajadores no precarios. Su condición de sobrevivencia, se puede considerar como de pobreza laboral extrema (Gráfico $\mathrm{N}^{\mathrm{o}} 7$ ).

\section{- $\quad$ Precariedad media ${ }^{10}$}

Una sexta parte del total de fuerza de trabajo (14.8\%) empleada en la RMG, labora bajo condiciones de precariedad media. Es decir, del total de fuerza de trabajo regional 14.8 personas laboran bajo condiciones de precariedad media. La estructura laboral de las actividades económicas con precariedad media, que registran un promedio superior al regional son tres: servicios diversos (31.1\%); construcción $(22.6 \%)$ restaurantes y servicios de alojamiento (20.7\%). Por debajo del promedio regional

8. Bajo estas condiciones laborales, la fuerza de trabajo recibe entre 4 y más de 5 salarios mínimos (SM), suficientes para adquirir la canasta alimenticia requerida (CAR): devengar un nivel salarial de 3.1 SM (para una explicación amplia de esta consideración véase Parra y Gámez, 2015). El total de la fuerza de trabajo con empleo cuenta con: prestaciones sociales, acceso a las instituciones de salud y, cuenta con un contrato de trabajo por tiempo indefinido.

9. En este nivel de precariedad, la fuerza de trabajo percibe entre menos de 1 hasta 3 salarios mínimos insuficientes para adquirir la CAR; no cuentan con acceso a instituciones de salud, no reciben prestaciones laborales y su contratación es temporal o sin contrato escrito.

10. La fuerza de trabajo en condiciones de precariedad media, percibe entre menos de 1 hasta $3 \mathrm{SM}$; no cuenta con acceso a instituciones de salud o con prestaciones laborales, el tipo de contrato es de inestabilidad: temporal o sin contrato escrito. 
se encuentran las restantes siete actividades económicas: comercio $(14.2 \%)$; servicios profesionales, financieros y corporativos (11.5\%); industria manufacturera $(10.8 \%)$; servicios sociales $(9.1 \%)$; transportes y comunicaciones (8.1 por ciento); gobiemo y organismos internacionales (4.3\%); industria eléctrica (4.1\%) (Gráfico $\mathrm{N}^{\circ} 7$ ). En este segmento intermedio de precariedad, la fuerza de trabajo empleada sobrevive bajo condiciones de pobreza laboral media.

- Precariedad baja ${ }^{11}$

El $39.2 \%$ del total regional de la fuerza de trabajo labora bajo condiciones de precariedad baja. Es decir, 501,097 trabajadores y sus familias se reproducen bajo dichas condiciones de precariedad. Cabe señalar que seis de las actividades económicas registran un promedio superior al regional: transportes y comunicaciones $(51.8$ por cada 100 de su fuerza de trabajo, laboran en condiciones de precariedad baja); industria manufacturera ( 47.8 por cada 100); servicios profesionales, financieros y corporativos (46.7 por cada 100 de sus trabajadores); comercio ( 45 por cada 100); servicios sociales (41.5 por cada 100 ); gobierno y organismos internacionales ( 40.4 por cada 100$)$.

Por debajo del promedio regional se encuentran: restaurantes y servicio de alojamiento ( 27.6 por cada 100 ); industria de la construcción (26.9 por cada 100); servicios diversos (15.5 por cada 100); industria eléctrica (14.2 por cada 100) (Gráfico No 7 ).

Gráfico $N^{\circ}$ 7. Tasa de participación de la fuerza de trabajo por actividad económica, 2005-2015

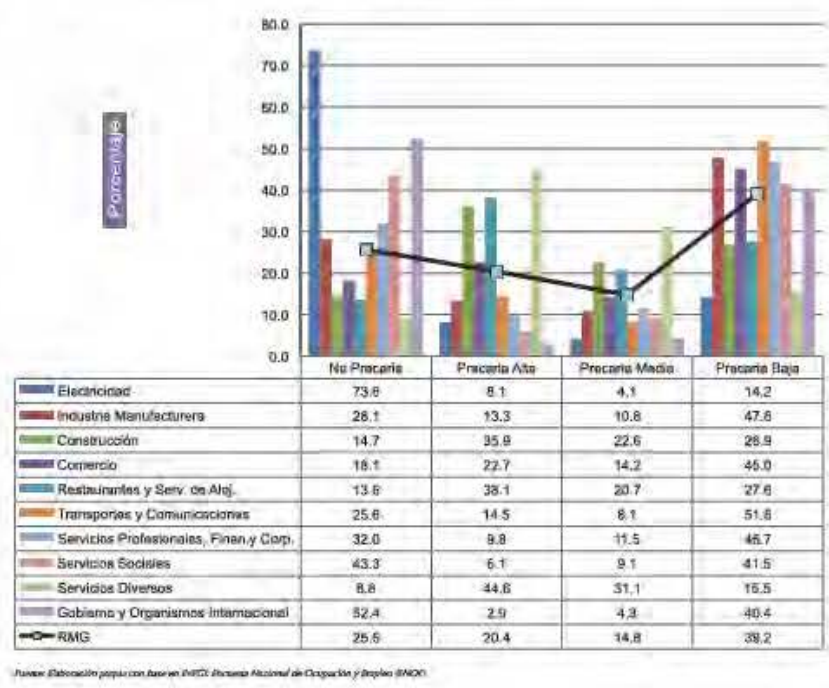

En suma, la precariedad baja constituye una de las estrategias más impulsadas para la compra de fuerza de trabajo por parte de los empleadores de la industria manufacturera, así como del comercio y los servicios públicos y privados.

\section{Consideraciones finales}

En 2015, la población de la RMG, registra un importante incremento en el nivel de ocupación, en un contexto de muy reducido crecimiento económico. menor tasa de desempleo, un estancamiento en la participación de la población en el mercado de trabajo. Este crecimiento anual, permite revertir la crisis de ocupación observada en 2014, y superar las consistentes dinámicas descendentes ocurridas en el periodo 2010-2013. Sin embargo, lo reiteramos, dicho incremento no cubre las necesidades de un puesto de trabajo requeridas por la población, como lo indica el estancamiento de la participación poblacional en el mercado de trabajo.

Un primer matiz, sobre el incremento en el nivel de la ocupación señala que la participación de los sujetos laborales es inestable y heterogénea en el tiempo, como lo muestra el comportamiento de la fuerza de trabajo y los trabajadores por su cuenta. Un segundo matiz, indica que la fuerza de trabajo sigue sosteniendo la mayor participación, de los sujetos laborales, en la estructura ocupacional de la RMG. Lo cual aunado a su cariz de compra-venta en el mercado, se constituye en un sujeto laboral de la mayor importancia, a escala nacional y regional, para analizarlo en relación con sus empleadores de cada una de las diez ramas de actividad económica, en términos de su distribución como de las condiciones que estos últimos imponen para su compra.

Un primer matiz, en el comportamiento de la compraventa de la fuerza de trabajo son los encuentros (empleos) y desencuentros (desempleo) que esta enfrenta en 2015 y en general durante el periodo 2005-2015, con sus empleadores. Un segundo matiz, corresponde a la participación que ésta registra por cada una de las ramas de actividad económica destacando siempre la industria manufacturera como la que mayor fuerza de trabajo contrata pero sin alcanzar la tasa de participación de 2006. En ambos, aspectos se expresa la tendencia de inestabilidad y heterogeneidad.

Por otra parte, las condiciones de compra-venta de la fuerza de trabajo, en 2015, continúan propiciando una erosión salarial, un deterioro en las prestaciones 
laborales y en el acceso a las instituciones de salud, así como un incremento en los contratos temporales y en la subordinación sin contratos escritos. Lo cual produce que tres cuartas partes de la fuerza de trabajo se reproduzcan bajo distintas formas de precariedad laboral. De suerte que un segmento tiende a enfrentar condiciones de pobreza laboral extrema, otro, de pobreza media y un tercero, de pobreza laboral baja.

\section{Bibliografía}

- Antunes, Ricardo (2012), Prefacio del texto de Adrián Sotelo Valencia, Los rumbos del Trabajo. Superexplotación y precariedad social en el siglo XXI, MA Porrúa-UNAM.

- Alves, Giovanni (2007), Dimensoes da reestrturação productiva. Ensaios de soiologís do trabalho, São Paulo, Editora Praxis.

- García Guzmán, Brígida (2010), “Inestabilidad laboral en México: el caso de los contratos de trabajo”. Estudios Demográficos y Urbanos, vol. 25, núm. 1, enero-abril, pp. 73-101. El Colegio de México, A.C. (2009).

- "Precariedad Laboral y Desempleo en México, 20002009". Ponencia presentada en la sesión especial "La participación económica en el contexto actual de crisis económica en América Latina", X Reunión Nacional de Investigación Demográfica en México, organizada por la Sociedad Mexicana de Demografía.

- Gaxiola Robles Linares, Sergio Cuauhtémoc (2013), "Análisis comparativo de la precariedad laboral en las tres principales metrópolis mexicanas para el 2010”, Revista Facultad de Ciencias Económicas: Investigación y Reflexión, Vol. XXI (1), Universidad Militar Nueva Granada, Bogotá, Colombia, Junio, 131-146.

- Gómez Solórzano Marco Augusto (2007) "La precariedad siempre existente”, en Mabel Burin, MarIa Lucero Jiménez Guzmán, Irene Meler (compiladoras), Precariedad laboral y crisis de la masculinidad. Impacto sobre las relaciones de género. CUCES, Argentina, 2007.

- Guadarrama, Rocío, Alfredo Hualde y Silvia López (2014), La Precariedad Laboral en México. Dimensiones, dinámicas y significados, UAM-El Colegio de La Frontera Norte.

- Hualde y Guadarrama (2012) "Precariedad laboral y heterogeneidad ocupacional: una propuesta teóricometodológica", Revista Mexicana de Sociología 74, núm. 2 (abril-junio, 2012).

- INEGI (2016) Encuesta Nacional de Ocupación y Empleo (ENOE), www.inegi.org.mx, consultado el 17 de febrero de 2016.

- Parra Ruiz, José María y Emilia Gámez F. (2015), “Crisis Laboral en la Región Metropolitana Guadalajara”, Revista Población y Desarrollo. Argonautas y Caminantes, Volumen 11, 2015 (2006).
- Parra Ruiz, José María y Emilia Gámez F. (2006), "Movilidad espacial de los trabajadores que laboran en la industria electrónica de la región metropolitana Guadalajara" Carta Económica Regional, núm. 91, Universidad de Guadalajara, enero-marzo, pp. 51-69, Guadalajara, Jalisco, México.

- Pries, Luger (2000), “Teoría sociológica del mercado de trabajo" en Enrique de la Garza (compilador), Tratado Latinoamericano de Sociología del Trabajo, ColmexUAM-FCE.

- Rojas García, Georgina y Carlos Salas Páez (2008), "La precarización del empleo en México, 1995-2004," Revista Latinoamericana de Estudios del Trabajo, segunda época, año 13, número 19, pp. 39-78.

- Sotelo Valencia, Adrián (2012), Los rumbos del Trabajo. Superexplotación y precariedad social en el siglo XXI, M.A. Porrúa-UNAM. 